\title{
An omic approach to spinal cord injury mechanisms
}

\author{
Erna A. van Niekerk*
}

Department of Neurosciences-0626, University of California, San Diego, La Jolla, CA 92093

\section{Article Info}

\section{Article Notes}

Received: August 04, 2016

Accepted: September 16, 2016

\section{*Correspondence:}

Dr. Erna A. van Niekerk, Ph.D.

Department of Neurosciences-0626, University of California-San Diego, 9500 Gilman Drive

La Jolla, CA 92093-0626, Telephone: (858) 534-8857, Fax: (858) 534-5220

Email: erna@ucsd.edu

(c) 2016 van Niekerk EA. This article is distributed under the terms of the Creative Commons Attribution 4.0 International License

\begin{abstract}
Spinal cord injury $(\mathrm{SCl})$ research continues to make substantial progress in identifying both neuron-intrinsic and neuron-extrinsic mechanisms that limit central nervous system (CNS) plasticity and regeneration. The identification of these mechanisms has in turn led to several novel strategies for therapeutically enhancing recovery of the injured CNS. Despite this progress, clinical translation remains a challenge for several reasons, including: 1 ) problems in projecting beneficial outcomes from small animal models to primate systems, 2) a lack of robust improvement in functional outcomes in animal models, and 3) difficulty replicating published reports in the field. Collectively, while the field has seen great progress, reconstructing the exquisite circuitry of the injured human CNS will require yet greater progress in both understanding of basic mechanisms underlying axonal growth and guidance, and testing of optimized therapies in models more predictive of potential human benefit.
\end{abstract}

\section{Introduction}

Moving toward the goal of developing useful therapies for SCI, over the past few decades there has been great success in identifying multiple mechanisms that limit the plasticity and regeneration of injured adult axons. While this progress has been impressive and expansive, it has yet to achieve success in the clinical arena, even as an increasing number of novel and creative therapies undergo clinical translation. This is a prescient moment to review fundamental advances in our understanding of mechanisms underlying abortive regeneration in the adult CNS, and to chart a path forward that might optimize the potential to generate detectable benefits to humans in future SCI clinical trials.

Mechanisms of axonal growth and regeneration in the adult cns

Neural development teaches us that the extending axon both express and respond to a precisely orchestrated series of events that ultimately guide the growing axon to its target, leading to the formation of functional synapses and the elimination of inappropriate or dysfunctional synapses ${ }^{1}$. The developing environment, in turn, must express a set of extracellular matrix molecules, cell adhesion molecules, and cells that guide and respond to actively extending axons ${ }^{2}$. Thus, successful axonal extension and target finding during development occur as a consequence of an exquisite orchestration of signals, both intrinsic and extrinsic to the developing neuron. Perturbation of any of these events can disrupt the architecture of the mature CNS. 


\section{Intrinsic Mechanisms}

Following injury, cell-intrinsic growth programs are activated that lead to axon regeneration and new synapse formation. Identifying axonal growth-promoting genes in adult CNS neurons is the subject of active and extensive study, as most CNS neurons display limited regeneration. Successful axonal regeneration employs networks of interrelated genes concomitant to the activated state of the neuron, including transcription activators and functional networks related to growth-specific signaling pathways ${ }^{3-7}$. A recent study using RNA sequencing technology indicated that successfully regenerating peripheral neurons coordinately express genes within regeneration networks ${ }^{8}$. This coordinated regulation of molecules is considered a hallmark feature of successful regeneration. In contrast, CNS neurons upregulated discordant networks after injury, suggesting an orchestrated expression of networks is necessary for successful axon regeneration. Proteomic network analysis in axotomized retinal ganglion cells further identified new regulatory hubs involved in survival and regeneration, where these molecules did not show significance in sensory neurons ${ }^{9}$. Given the multiple mechanisms that limitadultCNS plasticity and regeneration, the discordant expression of signalling networks and the molecular signature of adult central neurons may be significant contributors to limited regeneration ${ }^{10}$.

\section{Extrinsic Mechanisms}

Because sites of SCI lack permissive substrates for axonal growth, a number of experimental approaches have placed substrates to support axonal attachment and growth in lesion sites: including fetal neurons ${ }^{11}$, bioengineered scaffolds ${ }^{12}$, sciatic nerve bridges ${ }^{13}$, and neural stem cells ${ }^{14}$ to name a few. Each of these methods establishes a physical matrix in an injury site that permits the attachment and growth of certain classes of host axons, including local spinal projections and some axonal systems projecting from the brainstem to the spinal cord. Corticospinal (CST) axons are among the host axonal systems that regenerate into neural stem cell grafts ${ }^{11}$, a finding of critical importance because this is the most important system in humans for voluntary motor control. CST axons only regenerate into neural stem cell grafts that are driven towards a caudal, spinal cord fate, suggesting the molecular blueprint established during development is maintained in adulthood.

These observations generate a set of conditions in which we can utilize modern techniques of neuroscience to identify an unprecedented level of detail of the mechanisms by which adult neurons orchestrate communication with its permissive environment after injury to activate developmentally established growth mechanisms.

\section{Methods of discovery}

Due to the complexity of coordinated signalling and communication between cells wherein most events do not function in isolation, a systems level analysis based on transcriptomic, proteomic, and connectomic mechanisms is needed ${ }^{15}$. An omic analysis can be described as follow: macromic where big-data analytics reveal neural tract adaptation in response to injury and regeneration and system wide motor-sensory adaptation; mesomic where the connectome of newly formed synapses are mapped as structure-function models, revealing new circuitry between the regenerating axon and grafted substrates in the lesion site; micromic where the molecular identity of regenerating neurons and permissive substrates are established through the utilization of transcriptomic and proteomic network analysis. We are only now beginning to understand micromic analysis. Identifying causal pathways linking proteomic events to transcriptional states will allow us to more precisely determine the pathophysiology of a successful regenerating axon. Further connecting genomic perturbations to gene expression changes will allow us to better understand the limitations of an adult CNS axon and how to manipulate its growth state.

\section{Conclusion}

Great progress has been made in understanding basic mechanisms underlying the refractory state of axonal plasticity and regeneration after adult CNS injury. The identification of significant regulators of axonal growth will most likely be revealed through a systems level omics approach and genetic animal models. The next several years are subject to yield testing of top candidates, or combinations of candidate genes, to enhance the intrinsic growth state of the injured adult CNS neuron. Moreover, given the multiple mechanisms that converge to limit adult CNS plasticity and regeneration after injury, combination therapies will be required to measurably improve human outcomes after SCI; more research emphasis on combination approaches is needed. Finally, translation to the clinical arena may anticipate most success when a candidate therapy, or "set" of combination therapies, meets the criteria of: 1) validation in a severe rodent contusion model, 2) demonstration of efficacy in a larger animal model of regeneration, and 3) clear replication by independent investigators. The field awaits development of such therapies.

\section{References}

1. Tessier-Lavigne M, Goodman CS. The molecular biology of axon guidance. Science. 1995; 274(5290):1123-33.

2. Kiryushko D, Berezin V, Bock E. Regulators of neurite outgrowth: role of cell adhesion molecules. Ann N Y Acad Sci. 2004; 1014:140-54.

3. Park KK, Liu K, Hu Y, Smith PD, Wang C, Cai B. et al. Promoting axon regeneration in the adult CNS by modulation of the PTEN/mTOR pathway. Science. 2008; 322(5903):963-6.

4. Sun F, Park KK, Belin S, Wang D, Lu T, Chen G, et al. Sustained axon regeneration induced by co-deletion of PTEN and SOCS3. Nature. $2011 ; 480(7377): 372-5$ 
5. Fu Q, Hue J, Li S. Nonsteroidal anti-inflammatory drugs promote axon regeneration via RhoA inhibition. J Neurosci. 27(15):4154-64.

6. Cheah M, Andrews MR, Chew DJ, Moloney EB, Verhaagen J, Fässler $\mathrm{R}$, et al. Expression of an Activated Integrin Promotes Long-Distance Sensory Axon Regeneration in the Spinal Cord. J Neurosci. 2016; 36(27):7283-97.

7. Omura T, Omura K, Tedeschi A, Riva P, Painter MW, Rojas L, et al Robust Axonal Regeneration Occurs in the Injured CAST/Ei Mouse CNS. Neuron. 2015; 86(5):1215-27.

8. Chandran V, Coppola G, Nawabi H, Omura T, Versano R, Huebner EA, et al. A Systems-Level Analysis of the Peripheral Nerve Intrinsic Axonal Growth Program. Neuron. 89(5):956-70.

9. Belin S, Nawabi H, Wang C, Tang S, Latremoliere A, Warren P, et al. Injury-induced decline of intrinsic regenerative ability revealed by quantitative proteomics. Neuron. 2015; 86(4):1000-14.

10. van Niekerk EA, Tuszynski MH, Lu P, Dulin JN. Molecular and Cellular Mechanisms of Axonal Regeneration After Spinal Cord Injury. Mol Cell Proteomics. 2016; 15(2):394-408.
11. Kadoya K, Lu P, Nguyen K, Lee-Kubli C, Kumamaru H, Yao L, et al. Spinal cord reconstitution with homologous neural grafts enables robust corticospinal regeneration. Nat Med 22, 479 (May, 2016).

12. Gao M, Lu P, Bednark B, Lynam D, Conner JM, Sakamoto J, et al. Templated agarose scaffolds for the support of motor axon regeneration into sites of complete spinal cord transection. Biomaterials. 2013; 34(5):1529-36.

13. Houle JD, Amin A, Cote MP, Lemay M, Miller K, Sandrow H, et al. Combining peripheral nerve grafting and matrix modulation to repair the injured rat spinal cord. J Vis Exp. 2009; 20:(33).

14. Lu P1, Wang Y, Graham L, McHale K, Gao M, Wu D, et al. Long-distance growth and connectivity of neural stem cells after severe spinal cord injury. Cell. 2012; 150(6):1264-73.

15. Putin E, Mamoshina P, Aliper A, Korzinkin M, Moskalev A, Kolosov A, et al. Deep biomarkers of human aging: Application of deep neural networks to biomarker development. Aging (Albany NY). 2016; 8(5):1021-33. 